\title{
Microcarcinoma da Tireóide: Experiência e Conduta em Clínica Privada
}

\section{revisão}

\section{Celso U.M. Frigugletti Marco Aurélio V. Kulcsar}

\section{Cirurgia de Cabeça e Pescoço, Faculdade de Medicina Universidade Santo Amaro - UNISA, São Paulo, SP.}

Recebido em 18/12/06 Aceito em 04/01/07

\section{RESUMO}

Objetivo: Estudar o microcarcinoma papilífero observado nas tireoidectomias realizadas em nossa clínica privada. Material e métodos: Revisamos clínica e histologicamente 1.930 pacientes submetidos a tireoidectomias no período de 2002 a 2006, tendo 606 carcinomas e, desses, 332 como microcarcinomas papilíferos. Avaliamos sexo, idade, tipo histológico, tamanho da neoplasia, multifocalidade, freqüência do esvaziamento cervical, resultados da $\mathrm{PCl}$ e tireoglobulina sérica. Resultados: Dos 332 pacientes, 48 eram do sexo masculino e 146 tinham idade superior a 45 anos. Todos foram submetidos a tireoidectomia total, 19 concomitantemente a esvaziamento cervical de necessidade $(5,72 \%)$, e 313 a iodoterapia (94,27\%). Tivemos metástase a distância em 5 pacientes (1,5\%). Após 1 ano, de 170 pacientes submetidos à $\mathrm{PCl}$, temos 141 considerados livres de doença, $21 \mathrm{com}$ anti-tireoglobulina positivo, $6 \mathrm{com}$ tireoglobulina superior a $2 \mathrm{ng} / \mathrm{ml}$, sendo $1 \mathrm{com}$ metástase pulmonar e outro com recidiva linfonodal $(0,3 \%)$. Conclusão: Recomendamos que seja realizada a tireoidectomia total, esvaziamento cervical de necessidade e iodoterapia ablativa na presença de fatores clínicos e anátomo-patológicos de risco. (Arq Bras Endocrinol Metab 2007;51/5:774-782)

Descritores: Tireóide; Carcinoma papilífero; Microcarcinoma; Conduta clínica

\section{ABSTRACT}

Thyroid Microcarcinoma: Experience and Management in Private Clinic. Objective: The study of papillary microcarcinoma observed during the thyroidectomies in our private clinic. Material and methods: We reviewed clinical and histopathological results of 1,930 consecutive patients submitted for thyroidectomies in the period from 2002 to 2006, 606 had thyroid carcinomas but only 332 were papillary microcarcinoma. We studied gender, age, histologic type, size, multifocality, frequency of neck dissection, and results of Whole Body Scan and thyroglobulin. Results: Overall, we found 48 males and 146 older than 45 years old. Therapy included total thyroidectomy for all, therapeutic neck dissection in 19 (5.72\%), and radioiodine for $313(94.27 \%)$. We found 5 patients with distant metastases (1.5\%). After 1 year, from 170 submitted to WBS, we consider 141 free of disease, 21 have positive thyroglobulin antibody, 6 have thyroglobulin level above $2 \mathrm{ng} / \mathrm{ml}$, being 1 with lung metastases and other with lymph node recurrence $(0.3 \%)$. Conclusion: We recommend total thyroidectomy, therapeutic neck dissection and radioiodine ablation when present clinical and pathological risk factors. (Arq Bras Endocrinol Metab 2007;51/5:774-782)

Keywords: Thyroid; Papillary carcinoma; Microcarcinoma; Clinical approach 
$\mathrm{O}$ MICROCARCINOMA DA TIREÓIDE, definido pela OMS como aquele menor de $1 \mathrm{~cm}$, é achado freqüentemente nas peças cirúrgicas em concomitância com doenças benignas da tireóide e no rastreamento de nódulos realizado tanto por endocrinologistas como por outros especialistas. E indolente, raramente apresenta metástase a distância e causa morte; porém, existem casos mais agressivos, com metástases, e evolução desfavorável. Até o momento não conseguimos distinguir os indolentes dos agressivos, nem mesmo com marcadores moleculares. Revisamos nossa experiência em clínica privada e propomos tireoidectomia total, esvaziamento cervical quando houver metástase evidente e iodoterapia na presença de fatores clínicos e anátomo-patológicos de risco.

\section{MATERIAL E MÉTODOS}

De janeiro de 2002 a setembro de 2006, 1.930 pacientes foram submetidos a tireoidectomia pelos autores, oriundos de clínica privada. As cirurgias foram indicadas devido à suspeita de malignidade, hipertireoidismo, compressão de estruturas vizinhas ou em casos de bócio mergulhante. Destes, 606 pacientes tiveram diagnóstico de carcinoma, sendo 332 microcarcinomas papilíferos. Foram tratados por tireoidectomia total extracapsular (TT), esvaziamento cervical de forma terapêutica (ECT) nos casos de carcinoma papilífero, esvaziamento cervical de forma eletiva (ECE) nos casos de carcinoma medular; sempre com uso do exame de congelação intra-operatória para verificar tipo histológico e avaliar eventuais linfonodos presentes no compartimento central (nível VI, para- e pré-traqueal).

Foram encaminhados para pesquisa de corpo inteiro (PCI), preparados sem reposição hormonal por 3 a 4 semanas ou reposição de T3 por 2 semanas, seguido de 2 semanas sem qualquer hormônio, até obtenção de TSH maior que $30 \mathrm{U} / \mathrm{ml}$, e conjuntamente foram dosados tireoglobulina (TG) e anti-tireoglobulina (anti-TG).

Indicou-se iodoterapia sempre quanto presente algum fator de risco como nódulo maior de $1,5 \mathrm{~cm}$, sexo masculino, idade superior a 45 anos, metástase cervical ou a distância, tipo histológico agressivo, e captação maior que $1 \%$. A radioiodoterapia (IT) foi realizada por serviços especializados, que dependendo de sua rotina decidiram a dose ideal para cada caso precedida ou não da PCI. Os pacientes receberam dose mínima de $100 \mathrm{mCi}$ quando o intuito foi ablação, e doses de 150 a $250 \mathrm{mCi}$ naqueles casos que apresentavam metástase ou grande percentual de captação.
Foram reencaminhados aos seus endocrinologistas para acompanhamento em conjunto. Receberam tratamento com levotiroxina em dose supressiva e foram avaliados através de dosagens de TSH, TG, antiTG trimestralmente. Solicitamos ultrasonografia cervical (US) no sexto mês de pós-operatório. Após o primeiro ano pós-operatório, repetimos a PCI, com mesma metodologia para a elevação do TSH endógeno, ou, quando possível, com o TSH recombinante (rhTSH), e associamos as mesmas dosagens séricas. Posteriormente, aumentamos o intervalo das dosagens para semestralmente e o US para anualmente ou quando identificada linfadenopatia suspeita.

Os microcarcinomas papilíferos evidenciados nesta casuística foram estratificados quanto a sexo, idade, tipo histológico, tamanho da neoplasia, multifocalidade, freqüência do esvaziamento cervical, PCI e TG.

Os dados foram descritos através das freqüências absolutas e relativas. A existência de associação entre variáveis qualitativas foi verificada através do teste qui-quadrado de Pearson. Nos casos de associação significativa, a identificação das categorias com distribuição significativamente diferente foi feita pela partição do qui-quadrado. $\mathrm{O}$ nível de significância adotado nos testes foi igual a $5 \%$. As análises foram realizadas no software SAS versão 9.1.

\section{RESULTADOS}

Dos 1.930 pacientes tratados cirurgicamente, 1.692 eram do sexo feminino e 238 do sexo masculino, a idade variou de 6 a 84 anos, com média de 46,5 anos, 1.324 tiveram diagnóstico de benigno e 606, de maligno. Dos 606 pacientes com diagnóstico de maligno, 576 eram do tipo papilífero, 20 foliculares, 4 Hürthle, 4 carcinomas medular e 6 possuíam apenas hiperplasia de células C. A distribuição do diagnóstico histológico conforme o sexo está representada na tabela 1 .

Os pacientes com quadro tóxico se apresentaram como nódulo autônomo em 2 casos, 57 com doença de Graves e 52 com bócio multinodular, totalizando 114 casos. Após o tratamento cirúrgico, tiveram diagnóstico de malignidade: 9 do grupo com doença de Graves e 8 naqueles com bócios multinodulares, perfazendo 15,78 e $15,38 \%$ respectivamente.

Estratificamos os 576 casos de carcinoma papilífero conforme tamanho da neoplasia e avaliamos presença de fatores de risco como sexo masculino, idade superior a 45 anos e presença de multifocalidade, representados na tabela 2 . Correlacionamos o tamanho da neoplasia com emprego do esvaziamento cervical 
Tabela 1. Distribuição do diagnóstico histológico quanto ao sexo.

\begin{tabular}{lccccc}
\hline & $\mathbf{n}$ & Fem & \% & Masc & $\%$ \\
TOTAL & 1930 & 1692 & 87,66 & 238 & 12,33 \\
BENIGNO & 1324 & 1184 & 89,42 & 140 & 10,57 \\
MALIGNO & 606 & 508 & 83,82 & 98 & 16,17 \\
Ca Papilífero & 576 & 487 & 84,54 & 89 & 15,45 \\
Ca Folicular & 20 & 16 & 80 & 4 & 20 \\
Ca Hürthle & 4 & 3 & 75 & 1 & 25 \\
HP céls C & 6 & 2 & 33,33 & 4 & 66,66 \\
\hline
\end{tabular}

Tabela 2. Relação entre tamanho da neoplasia e fatores de risco.

\begin{tabular}{|c|c|c|c|c|c|c|c|}
\hline cm & $\mathbf{n}$ & Masc & $\%$ & $>45 a$ & $\%$ & Multifocal & $\%$ \\
\hline até 0,5 & 136 & 22 & 16,17 & 55 & 40,44 & 24 & 17,64 \\
\hline $0,6-1,0$ & 196 & 26 & 13,26 & 91 & 46,42 & 40 & 20,4 \\
\hline $1,1-1,5$ & 133 & 16 & 12,03 & 44 & 33,08 & 23 & 17,29 \\
\hline $1,6-4,0$ & 105 & 26 & 24,76 & 40 & 38,09 & 31 & 29,52 \\
\hline$>4,0$ & 6 & 4 & 66,66 & 4 & 66,66 & 1 & 16,66 \\
\hline TOTAL & 576 & 94 & 16,31 & 234 & 40,62 & 119 & 20,65 \\
\hline$p$ & & \multicolumn{2}{|c|}{0,0006} & \multicolumn{2}{|c|}{0,0976} & \multicolumn{2}{|c|}{0,1485} \\
\hline
\end{tabular}

Tabela 3. Relação entre tamanho da neoplasia e emprego do Esvaziamento Cervical Terapêutico.

\begin{tabular}{lccc}
\hline $\mathbf{c m}$ & $\mathbf{n}$ & Esvaziamento Terapêutico & $\%$ \\
até 0,5 & 136 & 6 & 4,41 \\
$0,6-1,0$ & 196 & 13 & 6,63 \\
$1,1-1,5$ & 133 & 16 & 12,03 \\
$1,6-4,0$ & 105 & 25 & 23,81 \\
$>4,0$ & 6 & 1 & 16,66 \\
TOTAL & 576 & 61 & 10,59 \\
p $<0,0001$ & & & \\
\hline
\end{tabular}

terapêutico (tabela 3). Apresentamos a PCI pós-operatória de acordo com o percentual de captação obtido na loja tireóidea e presença de metástase, e demonstramos sua relação com o nível de TG estimulada (tabela 4); porém, houve vários casos excluídos devido à falta de informação ou presença de anti-TG. A partir de 2005, tivemos menos casos excluídos, que constam na tabela 5. Encontramos 6 pacientes com metástase linfonodal cervical e 2 mediastinal, 5 pulmonares e 1 na bacia. Após 1 ano, realizamos 276 PCI, estimulada pelo TSH endógeno em 105 pacientes, pós nova dose terapêutica em 3 pacientes e nos demais 168 casos pósrhTSH; curiosamente, o rhTSH não elevou o TSH de forma suficiente em 11 pacientes.

Os 332 pacientes portadores de microcarcinoma papilífero foram estadiados, pela classificação de OHIO: 264 como I; 50, II; 13, III e 5 como IV, devido a 2 metástases em mediastino, $2 \mathrm{em}$ pulmão e 1 em bacia. A iodoterapia foi realizada em todos os pacientes classificados como II, III, IV e naqueles classificados como estádio I quando presente fator de risco, como 48 por serem do sexo masculino e 146 por possuírem idade superior a 45 anos, ou possibilidade de dificuldade no seguimento, como 166 por apresentar percentual de captação superior a $1 \%$, e 11 por presença de TG estimulada superior a $2 \mathrm{ng} / \mathrm{ml}$; portanto, apenas 19 pacientes não receberam IT. Após 1 ano, realizamos 170 PCI, estimulada pelo TSH endógeno em 69 pacientes, pós segunda dose terapêutica em 1 pacientes e nos demais 100 casos pós-rhTSH, neste último grupo obtivemos elevação insuficiente do TSH em 3 pacientes. Dos pacientes com metástase a distân- cia, apenas um paciente manteve a captação pulmonar. Obtivemos TG acima de 2 ng/ml em 6 casos; 4 foram estimuladas pelo TSH, 1 com a captação pulmonar mantida; e 2 pelo rhTSH, l deles comprovadamente com recorrência linfonodal no nível IV fora do compartimento central; e encontramos anti-TG positiva em 21 casos, $12,35 \%$ (tabela 6 ).

\section{DISCUSSÃO}

Recebemos de endocrinologistas, pacientes encaminhados com tireoidopatia já investigados com indicação cirúrgica ou, de outros especialistas, pacientes submetidos a rastreamento de nódulos, para avaliação e conduta. Sempre optamos por punção aspirativa guiada por ultrassom (PAAF) naqueles nódulos com risco como margens irregulares, vascularização intranodular, microcalcificações, independente de seu tamanho. Indicamos tratamento cirúrgico nos casos de nódulos com suspeita de neoplasia, em bócios mergulhantes ou com sintomas compressivos, nos casos de hipertireoidismo resistentes ao tratamento clínico ou na concomitância de hipertireoidismo com nódulos. $\mathrm{Na}$ avaliação pré-operatória, praticamente não usamos tomografia computadorizada, ressonância nuclear magnética ou PET-scan, pois não modificam a conduta cirúrgica.

Realizamos TT nos casos de bócio multinodular com envolvimento do parênquima posterior à veia tireóidea média bilateralmente, na presença de autoanticorpos e nos carcinomas independentemente de 
Tabela 4. $\mathrm{PCl}$ pós-operatória, relação com Tg estimulada $(\mathrm{ng} / \mathrm{ml})$, e presença de metástases.

\begin{tabular}{|c|c|c|c|c|c|c|c|}
\hline \% captação & $\mathbf{n}$ & TG $<2$ & TG $>2$ & Excluído & linfonodal & $\begin{array}{c}\text { Metástase } \\
\text { pulmão }\end{array}$ & bacia \\
\hline$<1$ & 329 & 220 & 44 & 65 & 3 & 4 & 1 \\
\hline $1,1-2$ & 67 & 35 & 13 & 19 & & & \\
\hline $2,1-3$ & 33 & 13 & 15 & 5 & 1 & & \\
\hline$>3$ & 33 & 17 & 8 & 8 & 4 & 1 & \\
\hline loja & 32 & 8 & 1 & 23 & & & \\
\hline TOTAL & 494 & 293 & 71 & 100 & 8 & 5 & 1 \\
\hline
\end{tabular}

Tabela 5. PCl pós-operatória e relação com Tg estimulada (ng/ml) após 2005.

\begin{tabular}{|c|c|c|c|c|c|c|c|c|c|}
\hline $\begin{array}{l}\text { \% } \\
\text { captação }\end{array}$ & $\mathbf{n}$ & TG $<2$ & $\%$ & TG $>2$ & $\%$ & $\begin{array}{c}\text { sem } \\
\text { informação }\end{array}$ & $\%$ & atTG + & $\%$ \\
\hline$<1$ & 110 & 72 & 65,45 & 21 & 19,09 & 4 & 3,63 & 13 & 11,8 \\
\hline $1,1-2$ & 27 & 10 & 37,03 & 13 & 48,14 & 2 & 7,4 & 2 & 7,4 \\
\hline $2,1-3$ & 15 & 5 & 33,33 & 8 & 53,33 & 0 & 0 & 2 & 13,3 \\
\hline$>3$ & 9 & 5 & 55,55 & 3 & 33,33 & 0 & 0 & 1 & 11,1 \\
\hline loja & 12 & 5 & 41,66 & 0 & 0 & 6 & 50 & 1 & 8,33 \\
\hline TOTAL & 173 & 97 & 56,09 & 45 & 26,01 & 12 & 6,93 & 19 & 11 \\
\hline
\end{tabular}

Tabela 6. Seguimento dos microcarcinomas após 1 ano.

\begin{tabular}{lcccc}
\hline & $\mathbf{n}$ & atTG + & atTG-/TG $>\mathbf{2}$ & Metástase \\
& 69 & 7 & 4 & 1 (pulmão) \\
PCI & 100 & 14 & 2 & 1 (linfonodo) \\
PCI PÓS THYROGEN & 1 & & 6 & 2 \\
PCI PÓS DOSE & 170 & 21 & & \\
TOTAL & & & & \\
\hline
\end{tabular}

seu tamanho $(1,2)$. Preferimos esta conduta, já que nossos índices de complicações definitivas nas TT são semelhantes aos da literatura e equivalentes ao das ressecções não totais, tanto para hipoparatireoidismo como paralisia nervo-recorrente; e além do que, reoperações de complementação para incidentalomas em bócios multinodulares podem evoluir com maior risco de complicações (3). Sabidamente nos casos de carcinoma, a TT permite exame anátomo-patológico de toda tireóide, remove todos os focos inclusive microscópicos, facilita o seguimento pela TG e previne recorrências.

A avaliação dos fatores de risco é fundamental para definirmos a conduta. Preferimos utilizar a classificação de Ohio, dentre os vários índices prognósticos existentes (tabela 7), que valoriza tanto o tamanho da neoplasia quanto a presença de multifocalidade, e em conjunto usamos índices que valorizam o sexo e idade do paciente como o TNM e o do Memorial Hospital (4-6). Encontramos significativo e progressivo percentual de indivíduos do sexo masculino, p de 0,0006, com aumento do tamanho da neoplasia; sem relação com idade superior a 45 anos, p de 0,0976 (tabela 2).

Mazzaferri (7) e Hay (8) demonstraram que o uso da TT em relação à ressecção não total no trata- mento de pacientes em estádios II e III diminui significativamente a recidiva e mortalidade. No estádio I, não existe diferença entre as duas técnicas; portanto, alguns preferem a ressecção não total devido a seu menor índice de complicações e possível manutenção de eutireoidismo, porém dificulta o seguimento com TG e em casos de recidiva, acarreta maior índice de complicações na reoperação devido a manipulaçaão de áreas com fibrose. Em nossa experiência, os pacientes submetidos à ressecção não total para doença benigna tiveram 59,3\% de hipotireoidismo, necessitando reposição hormonal $(1,2)$.

O microcarcinoma, definido pela WHO como menor que $1 \mathrm{~cm}$ (9), pode ser diagnosticado como: incidentaloma encontrado após tratamento de outras tireoidopatias (10); lesão oculta encontrada na investigação de metástase cervical; no rastreamento da forma familiar descrito por Lupoli (11); ou como diagnóstico inicial em apenas 4 a 12,6\% (12). Na maioria das vezes, seu tipo histológico é o papilífero e representa $30 \%$ de todos os carcinomas da tireóide (13).

Sua prevalência varia de 1,5 a $35,6 \%$, sendo que no Japão e na Finlândia excede 36\% (14). Em autópsias de adultos falecidos de causas não endocrinológicas foram identificados em $36 \%$ dos casos (15), e em 
Tabela 7. Classificação de Ohio.

\begin{tabular}{lcccc}
\hline & I & II & III & IV \\
TU $(\mathrm{cm})$ & $<1,5$ & $1,5-4$ & $>4$ & qq \\
MULTICÊNTRICO & $\tilde{n}$ & SIM & qq & qq \\
INVASÃO LOCAL & $\tilde{n}$ & $\tilde{n}$ & SIM & qq \\
META CERVICAL & $\tilde{n}$ & SIM & qq & qq \\
META DISTNCIA & $\tilde{n}$ & $\tilde{n}$ & $\tilde{n}$ & SIM \\
RECORRÊNCIA \% & 8 & 31 & 36 & 62 \\
ÓBITO \% & 0 & 6 & 14 & 65 \\
\hline
\end{tabular}

casuística brasileira, em 7,8\% (16). Alguns autores associam sua alta incidência com a qualidade do exame anátomo-patológico. Utilizamos grupo de patologistas que estudam de forma completa toda a glândula, o que eleva o achado de incidentalomas e multifocalidade. Infelizmente nosso levantamento retrospectivo não permitiu avaliarmos quais microcarcinomas eram incidentalomas e quais já possuíam diagnóstico prévio.

A grande controvérsia no tratamento cirúrgico do microcarcinoma está no uso da TT ou da lobectomia e istmectomia para lesões unifocais. Temos que lembrar que a multifocalidade varia de 20 a $40,7 \%$, e até $40 \%$ dos pacientes apresentam metástase cervical (13). Chow, ao estudar 203 pacientes com microcarcinoma, encontrou risco cinco vezes maior de metástase cervical na presença de multifocalidade (17). De uma forma geral, a recorrência ocorre em 3 a $5 \%$ dos pacientes, Baudin encontrou recorrência em lesões unifocais de $1,2 \%$ e de $8,6 \%$ nas lesões multifocais (18). Obtivemos presença de multifocalidade em $17,64 \%$ das lesões até com $0,5 \mathrm{~cm}$ e $20,4 \%$ naquelas de 0,6 a $1,0 \mathrm{~cm}$, sem diferença estatística $(\mathrm{p}=0,1485)$, e metástase cervical em 4,41 e $6,63 \%$, respectivamente (tabelas 2 e 3 ). Obviamente preferimos a TT, pois ao examinarmos toda tireóide, definimos a presença da multifocalidade, mudamos o estadiamento de I para II na classificação de Ohio, de tremenda importância na valorização dos resultados de recorrência e óbito, segundo Mazzaferri (tabela 7).

A significância clínica do microcarcinoma continua em debate. Devido à sua indolência, foi proposta nova terminologia de Carcinoma para Microtumor Papilar, naqueles casos comprovadamente únicos, sem envolvimento da cápsula tireóidea, sem subtipo histológico agressivo como células altas, que ocorrem em adultos com menos de 45 anos e que são achados incidentalmente $(15,19)$. Ito propõe normalmente observação e apenas recomenda tratamento cirúrgico naqueles localizados junto à traquéia, com possível invasão do nervo recorrente, PAAF sugerindo subtipos agressivos, ultrassom sugestivo de metástase cervical. Nos pacientes apenas acompanhados, 28,4\% foram tratados cirurgicamente devido a aumento do tamanho ou suspeita ultrassonográfica de metástase, o que confirma a importância da avaliação ultrassonográfica não só no pré-operatório, para definir possível necessidade de esvaziamento, mas também no seguimento (20-22). Em instituições como hospitais universitários, pode-se propor estudos prospectivos ou aplicar diferentes condutas desde observação até ressecções parciais, já que existe uma diminuição de responsabilidades, um desconhecimento pelo paciente de baixo nível intelectual de quem é seu verdadeiro médico; em clínica privada é completamente o oposto, atendemos pacientes diferenciados, na maioria das vezes com conhecimento adquirido em internet ou com amigos sobre a patologia, portanto temos a responsabilidade de indicar o tratamento mais completo disponível.

Nos pacientes tratados por bócio tóxico encontramos surpreendente freqüência de microcarcinomas, $15,78 \%$ nos pacientes com doença de Graves, muito além dos índices encontrados na literatura, 1 deles inclusive já apresentava metástase na bacia; felizmente todos apresentaram ótima evolução. Tal fato é questionado e relacionado a possível efeito do TRab $(23,24)$, o que nos faz atualmente indicar tratamento cirúrgico, TT, para todo paciente portador de Graves com nódulo.

Outra questão é quando o microcarcinoma alcança vasos linfáticos e dá metástase? Kasai mostrou que o diâmetro do microcarcinoma está relacionado com presença de metástase e propôs os termos "tiny" para lesão entre 5 e $10 \mathrm{~mm}$ e "minute" para lesão menor de $5 \mathrm{~mm}$, com diferentes incidências de metástase cervical, $59 \%$ e $13 \%$ respectivamente, e de extensão extratireóidea, $10 \%$ e 3\% (25). Da mesma forma, encontramos crescente necessidade de esvaziamento cervical com aumento do tamanho da neoplasia, $\mathrm{p}$ menor que 0,0001 (tabela 3 ).

O esvaziamento deve ser indicado eletivamente? Acreditamos que não, pois aumenta o hipoparatireoidsmo. Preferimos avaliar presença de linfonodos no compartimento central e lateral pela ultrassonografia pré-operatória. Observamos no intraoperatório toda região e cadeias júgulo-carotídeas, realizamos exame de congelação em eventuais linfonodos encontrados e, se confirmada metástase, preferimos o esvaziamento de todo compartimento central e não apenas "Picking procedure", afinal tal procedimento é incompleto e está associado a maior freqüência de recorrência $(26,27)$. A reabordagem do compartimento central, já manipulado, devido à recorrência, certamente eleva o percentual do hipoparatireodismo e principalmente o de lesão do nervo recorrente (28). Já 
foi comprovado que linfadenopatia metastática com rotura capsular apresenta pior prognóstico (29).

Em relação a marcadores moleculares prognósticos, apenas Sugitani correlacionou maior agressividade do microcarcinoma ao Ki67 e TGF $\beta$ (30), e Ito demonstrou que o microcarcinoma com metástase cervical apresenta aumento da imunoexpressão de ciclina D1 e Ki67, e diminuição p27 (31).

A IT tem valor? Especificamente para microcarcinomas estádio I é indicada seletivamente por alguns autores $(8,13,32)$, porém outros não demonstram nenhum efeito nas taxas de recorrência $(7,33)$. Na realidade, tais resultados se baseiam em trabalhos retrospectivos, e necessitamos dados prospectivos com seguimento prolongado $(34,35)$. Porém, ao realizarmos a TT deixamos o ligamento lateral de fixação da glândula à traquéia, chamado de Berry, preservado a fim de evitar lesão dos nervos recorrentes, o que mantém pequena quantidade de células evidenciáveis na PCI e com capacidade de produzir TG (figura 1), que podem ser facilmente destruídas pelo IT. Tal fato explica o achado de Schlumberger e Baudin de que ocorre melhora no percentual de TG indetectável quando utilizada iodoablação (36).

Em nosso meio, Ferraz e Brandão indicavam IT somente nos casos com captação superior a 6\% (37), provavelmente pela dificuldade em internar os pacientes devido à falta de leitos em serviços públicos e pelo fato de que com a progressão do processo de cicatrização ocorre fibrose do ligamento remanescen- te, com destruição destas células e queda progressiva da TG; mas sempre fica a incerteza se realmente obteremos níveis indetectáveis.

Realizamos PCI no pós-operatório com traçador de 2 a $5 \mathrm{mCi}$, mesmo nos casos com indicação para iodoterapia, para confirmarmos a radicalidade da TT. $\mathrm{Na}$ tabela 4 , aferimos a radicalidade dos autores, devido à captação menor de 1\% em 329 de 462 exames quantificados, portanto em 71,21\%. A grande crítica é o possível efeito de atordoamento das células ou efeito "stunning" existente; porém os resultados retrospectivos de Mazzaferri que norteiam mundialmente a conduta foram baseados em iodoterapia com PCI prévio, portanto também com atordoamento (4). O consenso europeu é contra tal prática rotineiramente, indica apenas em casos selecionados e obrigatoriamente fazem IT com $100 \mathrm{mCi}$, portanto semelhante à nossa conduta (38). Não discutiremos sobre doses. Sempre realizamos imagens pós-dose para confirmar captação pelo remanescente e avaliar diagnóstico de eventuais metástases com o maior traçador.

Nos pacientes com microcarcinoma sem nenhum fator de risco, com captação menor que $1 \%$, usamos o nível da TG estimulada pelo TSH para decidir a indicação de ablação (39); fato ocorrido em 21 pacientes $(19,09 \%)$, excluídos os 13 pacientes com presença de anti-TG positiva (11,8\%), dos 110 casos apresentados na tabela 5. O clube da tireóide de São Paulo propõe IT em todos os pacientes, visto que o paciente já passará pelo inconveniente hipotireoidismo
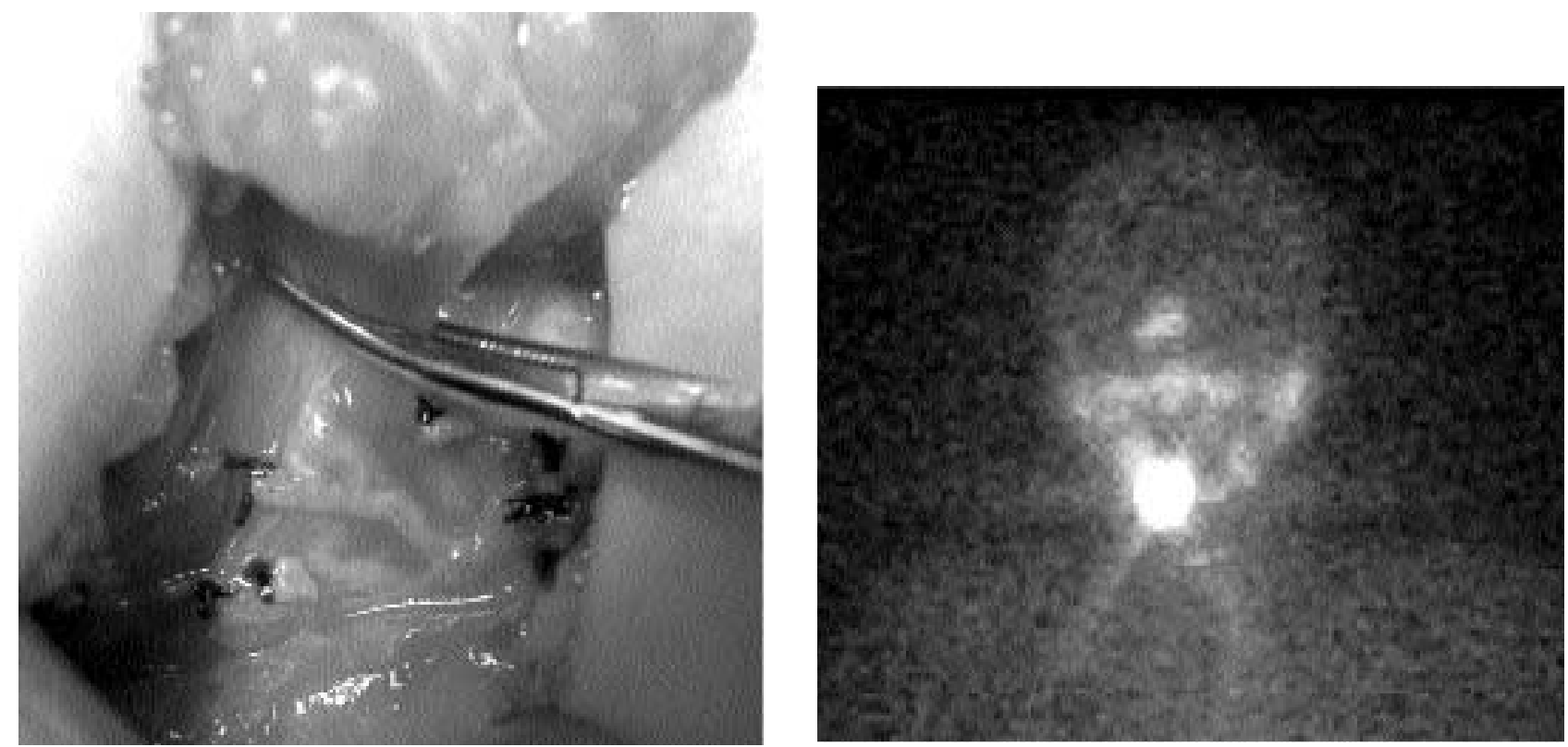

Figura 1. Ligamento de Berry preservado próximo ao nervo recorrente e sua captação. 
preparatório da PCI, pelo fato de ocorrer mínimo índice de complicações, e devido à inexistência de trabalhos prospectivos em pacientes com baixo risco (40).

No seguimento, solicitamos trimestralmente TG e TSH, bem como anti-TG para descartar falsos resultados (4l). Evitamos o US nos primeiros três meses de pós-operatório, para evitar encontro de imagens em loja tireoideana e linfonodos decorrentes do processo inflamatório e de cicatrização.

Após 1 ano realizamos PCI, apesar de questionável na literatura (42-45). Dependendo do seguro médico, nossos pacientes foram submetidos a preparos diferentes: sem T4 por quatro semanas, duas semanas com T3 e duas sem qualquer reposição e pós-rhTSH (46). Obviamente, o uso do rhTSH evita os desagradáveis sintomas do acentuado hipotireoidismo preparatório, porém implica num custo de R \$ 8.000,00 em comparação aos R\$ 500,00 da PCI clássica, o que inviabiliza seu uso por pacientes com seguros mais simples. Não sabemos porque em 11 pacientes não tivemos elevação suficiente do TSH pós-rhTSH, sendo, destes, 3 portadores de microcarcinomas; este fato nos desestimula em fracionar a dose do rhTSH no intuito de diminuir custos. Notamos que o preparo com T3 inicial é melhor tolerado pelo paciente e pode ser uma alternativa econômica, mas aguardamos estudo prospectivo comparativo, porém deve-se tomar grande cuidado na formulação do T3 para evitar superdosagens, ocorrida em l paciente.
Infelizmente não existe uniformidade do nível de TG estimulada no seguimento, principalmente devido a diferenças de padronização apesar do uso do consenso CRM 457 e devido ao tipo de estímulo, TSH endógeno ou rh-TSH $(47,48)$. Pellegretti considerou pacientes livres de doença quando apresentavam TG, na ausência de supressão, menor que 1 $\mathrm{ng} / \mathrm{ml}$, já que apresentavam recidiva menor de $1 \%$; em contrapartida a recidivas de $16 \%$ e $68 \%$ naqueles que apresentavam TG entre 1 e 10 e acima $10 \mathrm{ng} / \mathrm{ml}$, respectivamente. Outros consideraram, em pacientes de baixo risco tratados por TT e ablação, a TG menor que $2 \mathrm{ng} / \mathrm{ml}(42,45,48-52)$. Utilizamos TG estimulada pelo TSH ou rh-TSH abaixo de $2 \mathrm{ng} / \mathrm{ml} \mathrm{sem} \mathrm{inter-}$ ferência de anti-TG, PCI negativa e US negativo (visto que metástase cervical pode não elevar TG) para definir ausência de doença e inferir cura (53); portanto, daqueles com 1 ano completo de seguimento, temos 141 pacientes com microcarcinoma considerados curados (tabela 7). Dos demais 29, já acompanhados há mais de 1 ano, temos 1 com manutenção da metástase pulmonar e outro com recorrência linfonodal em programação de ECT.

As principais casuísticas existentes na literatura sobre microcarcinoma estão representadas na tabela 8 . A conduta é variável; quanto à tireoidectomia, 52,94\% preferem TT e $35,29 \%$, a ressecções não totais; quanto ao esvaziamento, $41,17 \%$ não indicam, $23,52 \%$

Tabela 8. Principais casuísticas sobre microcarcinoma.

\begin{tabular}{|c|c|c|c|c|c|c|c|c|}
\hline & \multirow[t]{2}{*}{$\mathbf{n}$} & \multicolumn{2}{|c|}{ Metástase } & \multirow{2}{*}{$\begin{array}{c}\text { Multifoco } \\
\%\end{array}$} & \multirow{2}{*}{$\begin{array}{c}\text { Recidiva } \\
\%\end{array}$} & \multirow{2}{*}{$\begin{array}{c}\text { Óbito } \\
\%\end{array}$} & \multirow{2}{*}{$\begin{array}{c}\text { Seg } \\
\text { anos }\end{array}$} & \multirow[t]{2}{*}{ Conduta } \\
\hline & & Iinf $\%$ & Dist $\%$ & & & & & \\
\hline WADA & 259 & 64,1 & 0 & & & 0 & 5 & LT/ECE \\
\hline HAY & 535 & 32 & 0,2 & 20 & 6 & 0,4 & 0,4 & TST \\
\hline NOGUCHI & 867 & 8,99 & 0,1 & 10 & 1,38 & 0,2 & 12 & TST \\
\hline FURLAN & 113 & 16,8 & 0,9 & 31,9 & 2,7 & 0 & 4,5 & $\mathrm{TST} / \mathrm{I}$ \\
\hline MIKI & 38 & 40 & & 26,7 & & 0 & 5 & TST/ECE \\
\hline BAUDIN & 207 & 43 & 3,8 & 40 & 3,9 & & 7 & LTouTT \\
\hline LIN & 97 & 18,5 & 6,2 & & & 1 & 6 & TT \\
\hline ROSARIO & 78 & 7,69 & 1,28 & 19,23 & & & 6,8 & TT \\
\hline ITO & 600 & 44,2 & & & & & 4 & TT \\
\hline CHEEMA & 74 & 16 & & & 8 & & 11 & $\mathrm{TT} / \mathrm{I}$ \\
\hline ROTI & 243 & 13 & 1,6 & 32 & & & 5 & $\mathrm{TT} / \mathrm{I}$ \\
\hline PELIZZO & 403 & 12,4 & 0,24 & 14,88 & 5,9 & 0 & 8 & TT/I \\
\hline FRIGUGLIETTI & 332 & 5,72 & 1,5 & 19,27 & 0,3 & 0 & & $\mathrm{TT} / \mathrm{ECT} / \mathrm{I}$ \\
\hline ROSEN & 99 & 29,29 & 0,3 & 15,15 & 8 & 0,1 & 5 & TT/ECE/I \\
\hline LEE & 302 & 29,7 & 0 & 16,3 & & & & TT/ECE/I \\
\hline PELLEGRITI & 187 & 24,6 & 2,7 & 26,7 & 14,4 & 0 & 4 & $?$ \\
\hline SUGITANI & 190 & 17,89 & 0,015 & & 5,78 & 2,1 & 10 & $?$ \\
\hline TOTAL & 4624 & 24,93 & 1,34 & 19,43 & 5,63 & 0,56 & & \\
\hline
\end{tabular}

$\mathrm{LT}$, lobectomia total

TST, tireoidectomia subtotal

TT, tireoidectomia total

ECE, esvaziamento cervical eletivo

$E C T$, esvaziamento cervical terapêutico ou de necessidade

I, iodoterapia 
propõem ECE e 5,88\% ECT; quanto a IT, temos indicação em 41,17\% e sem indicação em 47,05\%. Comparativamente, temos índices de multifocalidade e metástase a distância semelhantes, mas percentual de metástase linfonodal $(5,72 \%)$ menor que a média de $24,93 \%$, provavelmente elevada pelas casuísticas japonesas que indicam esvaziamento cervical eletivo $(8,12,18,21,26,30,54-60)$.

Concluímos que o microcarcinoma é diagnosticado cada vez mais, devido a rastreamentos com US e pela incessante procura em peças cirúrgicas pelos patologistas. Normalmente são indolentes e sem risco de mortalidade. Vários se apresentam em estádios mais avançados devido à presença de fatores de risco como sexo masculino, idade superior a 45 anos, multifocalidade e metástase cervical. Indicamos a TT, esvaziamento de necessidade do compartimento central e IT. Devemos buscar promissores marcadores moleculares, de uso prático, que definam prognóstico a fim de selecionar para cada paciente o melhor tratamento.

\section{REFERÊNCIAS}

1. Friguglietti CUM, Lin CS, Kulcsar MAV. Total thyroidectomy for benign thyroid disease. Laryngoscope 2003;113 (10):1820-6.

2. Giles $Y$, Boztepe $H$, Terzioglu T, Tezelman S. The advantage of total thyroidectomy to avoid reoperation for incidental thyroid cancer in multinodular goiter. Arch Surg 2004;139:179-82.

3. Mazzaferri EL, Kloos RT. Clinical review 128: current approach to primary therapy for papillary and follicular thyroid cancer. J Clin Endocrinol Metab 2001;86:1447-62.

4. Shaha AR, Shah JP, Loree TR. Risk group stratification and prognostic factors in papillary carcinoma of thyroid. Ann Surg Oncol 1996;3:534-8.

5. Shah JP, Kian K, Forastiere A, Garden A, Hoffman HT, Jack Lee J, et al. American Joint Committee on Cancer. Cancer staging manual. 6th edition. New York: Springer-Verlag, 2002. pp. 77-87.

6. Mazzaferri EL, Jhiang SM. Long-term impact of initial surgical and medical therapy on papillary and follicular thyroid cancer. Am J Med 1994:97:418-28.

7. Hay ID, Grant CS, Van Heerden JA, Goellner JR, Ebersold JR, Bergstralh EJ. Papillary thyroid microcarcinoma: a study of 535 cases observed in a 50-year period. Surgery 1992; 112:1139-47.

8. Hedinger C, Williams ED, Sobin LH. Histological typing of thyroid tumours. International histological classification of tumours. World Health Organization. 2nd ed. Berlin: Springer-Verlag, 1988. vol. 11.

9. Burguera B, Gharib H. Thyroid incidentalomas. Prevalence, diagnosis, significance, and management. Endocrinol Metab Clin North Am 2000;29:187-203.

10. Lupoli G, Vitale G, Caraglia M, Fittipaldi MR, Abbruzzese A, Tagliaferri $P$, et al. Familial papillary thyroid microcarcinoma: a new clinical entity. Lancet 1999;353:637-9.

11. Lin KD, Lin JD, Huang MJ. Clinical presentations and predictive variables of thyroid microcarcinomas with distant metastasis. Int Surg 1997;82:378-81.

12. Bramley MD, Harrison BJ. Papillary microcarcinoma of the thyroid. Br J Surg 1996;83:1674-83.

13. Harach HR, Franssila KO, Wasenius VM. Occult papillary carcinoma of the thyroid. A normal finding in Finland. A systematic autopsy study. Cancer 1985;56:531-8.
14. Zubair WB, LiVolsi VA. Microcarcinoma of the thyroid. Adv Anat Pathol 2006;13:69-75.

15. de Matos PS, Ferreira APC, Ward LS Prevalence of papillary microcarcinoma of the thyroid in Brazilian autopsy and surgical series. Endocr Pathol 2006:17(2):165-73.

16. Chow SM, Law SCK, Chan JKC, Aun SK, Yau S, Lau WH. Papillary microcarcinoma of the thyroid - prognostic significance of lymph node metastases and multifocality. Cancer 2003:98:31-40.

17. Baudin E, Travagli JP, Ropers J, Mancusi F, Bruno-Bossio G, Caillou B, et al. Microcarcinoma of the thyroid gland: the Gustave Roussy Institute experience. Cancer 1998; 83:553-9.

18. Rosai J, LiVolsi VA, Sobrinho-Simões M. Renaming papillary microcarcinoma of the thyroid gland: the Porto proposal. Int J Surg Pathol 2003;11:249-51.

19. Ito Y, Tomoda C, Uruno T, Takamura Y, Miya A, Kobayashi K, et al. Preoperative ultrasonographic examination for lymph node metastasis: usefulness when designing lymph node dissection for papillary microcarcinoma of the thyroid. World $\mathbf{J}$ Surg 2004;28:498-501.

20. Ito Y, Uruno T, Nakano K, Takamura Y, Miya A, Kobayashi K, et al. An observation trial without surgical treatment in patients with papillary microcarcinoma of the thyroid. Thyroid 2003;13:381-7.

21. Ito Y, Tomoda C, Uruno T, Takamura Y, Miya A, Kobayashi K, et al. Papillary microcarcinoma of the thyroid: How should it be treated? World J Surg 2004;11:1115-21.

22. Pellegriti G, Belfiore A, Guiffrida D, Lupo L, Vigneri R. Outcome of differentiated thyroid cancer in Graves' patients. J Clin Endocrinol Metab 1998;83:2805-9.

23. Stocker DJ, Burch HB. Thyroid cancer yield in patients with Graves' disease. Minerva Endocrinol 2003;28:205-12.

24. Kasai N, Sakamoto A. New subgrouping of small thyroid carcinomas. Cancer 1987:60:1767-70.

25. Noguchi S, Yamashita H, Murakami N, Nakayama I, Masakatsu, Kawamoto H. Small carcinomas of thyroid. A long-term follow-up of 887 patients. Arch Surg 1996;131:187-91.

26. Shaha AR. Management of the neck in thyroid cancer. Otolaryngol Clin North Am 1996;31:823-31.

27. Shindo M, Wu JC, Park EF, Tanzella F. The importance of central compartment elective lymph node excision in the staging and treatment of papillary thyroid cancer. Arch Otolaryngol Head Neck Surg 2006:132:650-4.

28. Yamashita H, Noguchi S, Murakami N, Toda M, Uchino S, Watanabe $S$, et al. Extracapsular invasion of lymph node metastasis. A good indicator of disease recurrence and poor prognosis in patients with thyroid microcarcinoma. Cancer 1999;86:842-9.

29. Sugitani I, Yanagisawa A, Shimizu A, Kato M, Fujimoto $Y$. Clinicopathologic and immunohistochemical studies of papillary thyroid microcarcinoma presenting with cervical lymphadenophaty. World J Surg 1998;22:731-7.

30. Ito Y, Uruno T, Takamura Y, Miya A, Kobayashi K, Matsuzuka $F$, et al. Papillary microcarcinomas of the thyroid with preoperatively detectable lymph node metastasis show significantly higher aggressive characteristics on immunohistochemical examination. Oncology 2005;68:87-96.

31. Sawka AM, Thephamongkhol K, Brouwers M, Thabane L, Browman G, Gerstein HC. A systematic review and metaanalysis of the effectiveness of radioactive iodine remnant ablation for well-differentiated thyroid cancer. J Clin Endocrinol Metab 2004;89:3668-76.

32. Appetecchia M, Scarcello G, Pucci E, Procaccini A. Outcome after treatment of papillary thyroid microcarcinoma. J Exp Clin Cancer Res 2002;21:159-64.

33. Mazzaferri E. A randomized trial of remnant ablation - In search of an impossible dream? J Clin Endocrinol Metab 2004;89:3662-4.

34. Dragoiescu C, Hoekstra OS, Kuik DJ, Lips P, Plaizier MA Rodrigus PT, et al. Feasibility of a randomized trial on adjuvant radio-iodine therapy in differentiated thyroid cancer. Clin Endocrinol 2003;58:451-5. 
35. Schlumberger M, Baudin E. Serum thyroglobulin determination in the follow-up of patients with differentiated thyroid cancer. Eur J Endocrinol 1988;138:249-52.

36. Ferraz AR, Brandão LG. Câncer da glândula tireóide. In: Brandão LG, Ferraz A (eds). Cirurgia de Cabeça e Pescoço. São Paulo: Ed. Roca, 1989. p. 620.

37. Pacini F, Schlumberger M, Dralle H, Elisei R, Smit JWA, Wiersinga W. European consensus for the management of patients with differentiated thyroid cancer of the follicular ephitelium. Eur J Endocrinol 2006;154:787-803.

38. Grünwald F, Menzel C, Fimmers R, Zamora PO, Biersack HJ. Prognostic value of thyroglobulin after thyroidectomy before ablative radioiodine therapy in thyroid cancer. J Nucl Med 1996;37:1962-4.

39. Ward LS, Marrone M, Camargo RY, Watanabe T, Tincani AJ, Assumpção LVM, et al. Câncer diferenciado da tiróide de baixo risco-revisão do estado atual da literatura e proposta de conduta. Arq Bras Endocrinol Metab 2006;50:550-7.

40. Spencer CA, Takeuchi M, Kazarosyan M. Serum thyroglobulin autoantibodies: Prevalence, influence on serum thyroglobulin measurement, and prognostic significance in patients with differentiated thyroid carcinoma. J Clin Endocrinol Metab 1998;83:1121-7.

41. Pellegriti G, Scollo C, Lumera G, Regalbuto C, Vigneri R, Belfiore A. Clinical behavior and outcome of papillary thyroid cancers smaller than $1.5 \mathrm{~cm}$ in diameter: study of 299 cases. J Clin Endocrinol Metab 2004;89:3713-20.

42. Cailleux AF, Baudin E, Travagli IP. Is diagnostic iodine-131 scanning useful after total ablation for differentiated thyroid cancer? J Clin Endocrinol Metab 2000;85:175-8.

43. Pacini F, Capezzone M, Elisei R, Ceccarelli C, Taddei D, Pinchera A. Diagnostic 131-iodine whole-body scan may be avoided in thyroid cancer patients who have undetectable stimulated serum Tg levels after initial treatment. J Clin Endocrinol Metab 2002;87:1499-501.

44. Schlumberger M, Berg G, Cohen O, Duntas L, Jamar F, Jarzab $B$, et al. Follow-up of low-risk patients with differentiated thyroid carcinoma: a European perspective. Eur J Endocrinol 2004;150:105-12.

45. Robbins RJ, Tuttle RM, Sharaf RN, Larson SM, Robbins HK, Ghossein RA, et al. Preparation by recombinant human thyrotropin or thyroid hormone withdrawal are comparable for the detection of residual differentiated thyroid carcinoma. $\mathbf{J}$ Clin Endocrinol Metab 2001;86:619-25.

46. Baloch Z, Carayon P, Conte-Devolx B, Demers LM, Feldt-Rasmussen U, Henry JF, et al. Laboratory medicine practice guidelines. Laboratory support for the diagnosis and monitoring of thyroid disease. Thyroid 2003;13:3-126.

47. Robbins RJ, Chon JT, Fleisher M, Larson SM, Tuttle RM. Is serum thyroglobulin response to recombinant human thyrotropin sufficient, by itself, to monitor for residual thyroid carcinoma? J Clin Endocrinol Metab 2002;87:3242-7.

48. Haugen BR, Pacini F, Reiners C, Schlumberger M, Ladenson PW, Sherman SI, et al. A comparison of recombinant human thyrotropin and thyroid hormone withdrawal for the detection of thyroid remnant or cancer. J Clin Endocrinol Metab 1999:84:3877-85.

49. Mazzaferri EL, Robbins RJ, Spencer CA, Braverman LE, Pacini $F$, Wartofsky $L$, et al. A consensus report of the role of serum thyroglobulin as a monitoring method for low-risk patients with papillary thyroid carcinoma. J Clin Endocrinol Metab 2003;88:1433-41.
50. Pacini F, Molinaro E, Lippi F, Castagna MG, Agate L, Ceccarelli $\mathrm{C}$, et al. Prediction of disease status by recombinant human TSH-stimulated serum Tg in the postsurgical followup of differentiated thyroid carcinoma. J Clin Endocrinol Metab 2001;86:5686-90.

51. Wartofsky L. Management of low risk well differentiated thyroid cancer based only upon thyroglobulin measurement after recombinant human thyrotropin. The rhTSH-Stimulated Thyroglobulin Study Group. Thyroid 2002;12:121-34.

52. Torlontano $M$, Attard M, Crocetti U, Tumino S, Bruno R, Costante G, et al. Follow-up of low-risk patients with papillary thyroid cancer: Role of neck ultrasonography in detecting lymph node metastases. J Clin Endocrinol Metab 2006;89:3402-7.

53. Wada N, Duh OY, Sugino K, Iwasaki H, Kameyama K, Mimura $\mathrm{T}$, et al. Lymph node metastasis from 259 papillary thyroid microcarcinomas: frequency, pattern of occurrence, and optimal strategy for neck dissection. Ann Surg 2003;237:399407.

54. Furlan JC, Bedard Y, Rosen IB. Biologic basis for the treatment of microscopic, occult well-differentiated thyroid cancer. Surgery 2001;130:1050-4.

55. Cheema Y, Olsen S, Elson D, Chen H. What is the biology and optimal treatment for papillary microcarcinoma of the thyroid? J Surg Res 2006;134:160-2.

56. Roti E, Rossi R, Trasforini G, Bertelli F, Ambrosio MR, Busutti $L$, et al. Clinical and histological characteristics of papillary thyroid microcarcinoma: results of a retrospective study in 243 patients. J Clin Endocrinol Metab 2006;91:2171-8.

57. Pelizzo MR, Boschin IM, Toniato A, Piotto A, Bernante P, Pagetta $C$, et al. Papillary thyroid microcarcinoma: prognostic factors, management and outcome in 403 patients. Eur J Surg Oncol 2006;32(10):1144-8.

58. Rosen IB, Azadian A, Walfish PG. Adverse aspects of small thyroid cancer and need for treatment. Head Neck 1995; 17:373-6.

59. Lee J, Rhee Y, Lee S, Ahn CW, Cha BS, Kim KR, et al. Frequent, aggressive behaviors of thyroid microcarcinomas in Korean patients. Endocr J 2006;3(5):627-32.

60. Miki H, Oshimo K, Inoue H, Kawano M, Tanaka K, Komaki K, et al. Diagnosis and surgical treatment of small papillary carcinomas of the thyroid. J Surg Oncology 1993;54:78-81.

61. Rosário PW, Fagundes TA, Purish S. Tratamento do microcarcinoma de tireóide. Arq Bras Endocrinol Metab 2004;48:855-60.

Endereço para correspondência:

Celso U.M. Friguglietti

Av. Paulista 1159, cj.1514

01311-200 São Paulo, SP

E-mail: frigugli@uol.com.br 